\title{
Time-dependent numerical simulation of diffraction and absorption effects in diagnostics of short high-power microwave pulses using wide-aperture liquid calorimeters
}

\author{
V.P. Tarakanov $^{1,2}$, A.I. Klimov ${ }^{3,4}$, I.V. Pegel ${ }^{3,4}$, P.V. Priputnev ${ }^{3}$, and E.M. Totmeninov ${ }^{3}$ \\ ${ }^{1}$ Joint Institute for High Temperatures, Russian Academy of Sciences, Moscow, Russia, karat@tarak.msk.su \\ ${ }^{2}$ National Research Nuclear University MEPhI, Moscow, Russia \\ ${ }^{3}$ Institute of High Current Electronics SB RAS, Tomsk, Russia, klimov@1fe.hcei.tsc.ru \\ ${ }^{4}$ National Research Tomsk Polytechnic University, Tomsk, Russia
}

\section{Introduction}

To measure the energy of high-power microwave pulses, calorimeters with disc-shaped wide-aperture absorbing loads filled with a working liquid based on ethanol are used $[1,2]$. Their principle of operation is based on an increase in the volume of the liquid due to the absorption of the energy of the microwave pulse. Using a microwave detector recording the envelope of the microwave pulse in conjunction with the calorimeter allows to determine the amplitude of the power in the pulse. The detector with a receiving antenna is usually installed behind the load - for example, to register a signal that has passed with attenuation through the absorbing liquid. In experiments $[3,4]$ it was found that the measured shape of the microwave signal depends on the registration point and can differ significantly from the observed in the absence of a calorimeter.

To figure out the origins of this dependence, numerical modeling using the axisymmetric (RZ) and planar (XZ) versions of the fully electromagnetic PiC-code KARAT [5] was performed. The parameters of the microwave pulses corresponded to the S-band as it was in experiments [4] using the SINUS-7 accelerator.

To take into account the frequency dispersion of the absorbing mixture, a Lorentz model implemented in the KARAT code was applied, based on the presentation of current in the medium by harmonic oscillators. The number of the oscillators with different eigenfrequencies and Q-factors, and their density, are selected in such a way that the dispersion curves known from the experiment can be approximated with a given accuracy.

The modeling demonstrated that, depending on the position of the registration point, the shape of the recorded pulse undergoes a distortion compared to that measured in the neck of the horn antenna, both because of the diffractive rounding of the calorimeter load by the electromagnetic wave, and because of the effect of frequencydependent spatial attenuation of the wave passing through the load.

\section{Diffraction and time-dependent interference of microwave field behind the calorimeter}

As a rule, a gap is left between the radiating horn antenna and the calorimeter, at least in order to mechanically separate the calorimeter from the microwave source (jitter transmitted from vacuum pumps or shaking from the operation of a pulsed magnetic system leads to a decrease in the sensitivity of the calorimeter and the accuracy of the microwave pulse energy measurement [1]).
The radiation that has passed through this gap, due to the diffraction at the lateral edge of the load of the calorimeter, falls into the region of the geometric shadow behind the calorimeter, where an interference pattern is formed. The amplitude of the diffraction field in this region can substantially exceed the amplitude of the wave transmitted with attenuation through the absorbing liquid. The simulations showed that, not only the amplitude of the detected signal depends on the position of the registration point in this region, but, more importantly, its shape (Fig. 1).

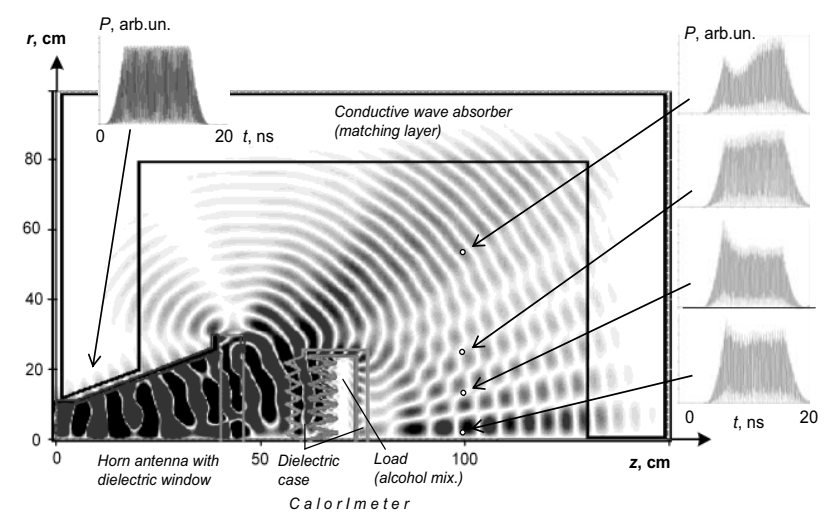

Fig. 1. Simulated $B_{\varphi}$ field pattern with a fundamental frequency of $3.8 \mathrm{GHz}$ in the vicinity of radiating horn antenna and the calorimeter load; waveforms of power flux density in the neck of the horn and at several points in a plane behind the calorimeter. The incident pulse has $16 \mathrm{~ns}$ full width, with $3 \mathrm{~ns}$ rise and fall

Distortion of the shape of the pulse is explained by the fact that the formation of the interference pattern at the front of the incident pulse and its destruction at the decay of the pulse behind the obstacle (the calorimeter) occurs with a delay determined by the difference in the arrival time of the diffraction waves from the edge of the obstacle to the observation point. Thus, distortion of the pulse shape takes place in off-axis regions, where the path difference is not zero. Maximally simplifying the situation, we can, instead of the obstacle in the form of a disk of some diameter, consider a flat strip of the same width, two edges of which will be the sources of cylindrical secondary waves.

Figure 2 shows examples of the envelopes of the "quadratic detector" signals obtained on the basis of the above elementary considerations, with a $2 \mathrm{~ns}$ variation in the wave path for the cases of in-phase and anti-phase addition of two secondary waves for a trapezoid initial pulse. As can be seen, the forms of the resulting pulses 
have the same characteristic features as those observed in the simulation.

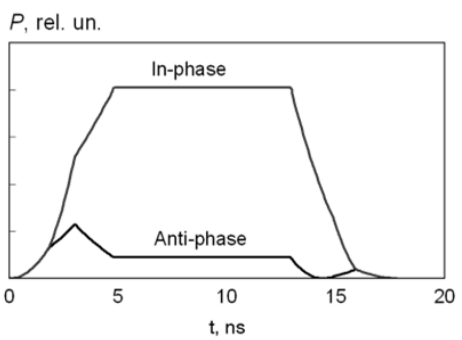

Fig. 2. An example of "quadratic" envelopes with in-phase and antiphase summation of diffraction fields of trapezium pulse duration of $16 \mathrm{~ns}$ with fronts of $3 \mathrm{~ns}$ at points with a path difference of $2 \mathrm{~ns}$

\section{Frequency-dependent decay of microwave pulses in the absorbing mixture}

It is possible to reduce the amplitude of the waves enveloping the calorimeter by means of its closer contact with the radiating antenna or by increasing the diameter of the calorimetric load. However, the pulse, which passes through the absorbing liquid with attenuation, can differ substantially in form from the incident pulse. This is due to frequency dependence of the microwave damping in the absorbing mixture. Indeed, even in the approximation of an absorbing medium with a constant conductivity independent of frequency, the spatial decrement of the wave (the skin depth) is inversely proportional to the square root of the frequency. As a result, if the wave decay at the fundamental frequency is strong (as is done in practice), then low-frequency components dominate in the pulse that reaches the rear side of the load, and its shape may radically differ from the original one.

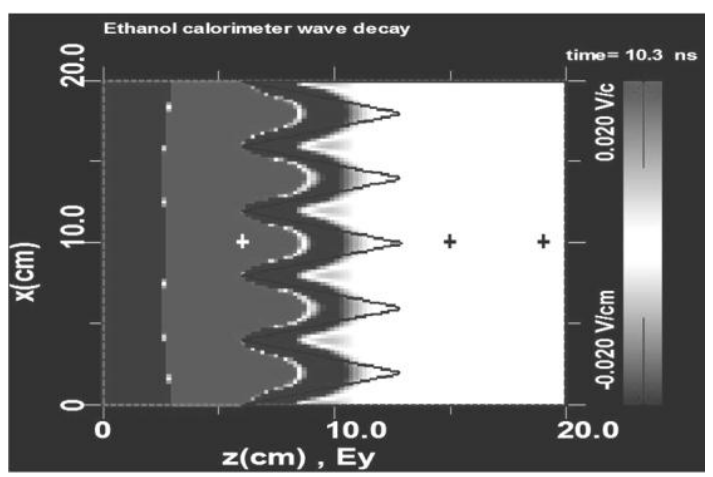

Fig. 3. The pattern of transverse component of the electric field when a plane wave falls on the ribbed surface of the absorbing medium of the calorimeter, simulated in planar (XZ) model. Pluses indicate control points, in which the field waveforms are monitored (Fig. 4)

The simulation confirmed that, with a given thickness of the absorbing medium, most significant distortions are experienced by those pulses that have steep fronts, and, respectively, a strongly "unbalanced" RF oscillation at this point. In this case, the sign of the lowfrequency ejection is determined by the initial (or final) phase of the RF oscillation (Fig. 4, a). Pulses in which the RF oscillations build up and decay smoothly, for example, a Gaussian pulse, are transmitted with relatively small low-frequency distortion (Fig. 4, c).

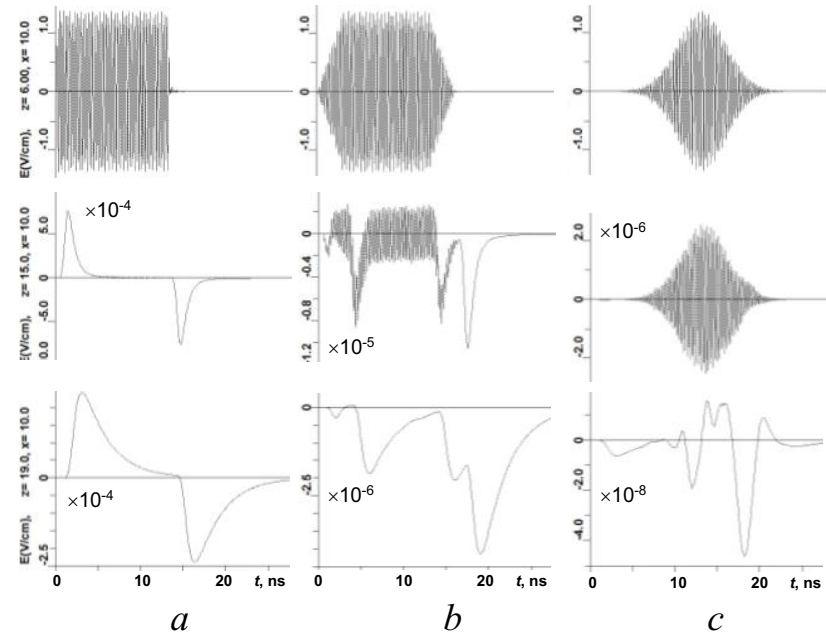

Fig. 4. Simulated waveforms of electric field at control points marked in Fig. 3: outside the absorbing mixture (top) and inside it (middle, bottom), for incident pulses of various shapes: $a$ - rectangular pulse containing an integer number of RF periods; $b$ - trapezoidal pulse; $c$ - pulse with Gaussian enveloping

\section{Conclusion}

Thus, the numerical modeling demonstrated that when registering the shape of the envelope of a nanosecond microwave pulse using a detector located behind the absorbing load of a wide-aperture liquid calorimeter, the shape of the detected signal under certain conditions may strongly differ from the pulse emitted by the microwave source. The corresponding limitations must be considered when performing such measurements.

\section{References}

1. Shkvarunets A.G. A broadband microwave calorimeter of large cross section // Instruments and Experimental Techniques, 1996, V. 39, Issue 4, pp. 535-538.

2. Vykhodtsev P.V., Elchaninov A.A., Klimov A.I., Kovalchuk O.B., Totmeninov E.M. Liquid calorimeters for measuring the energy of high-power microwave pulses // Instruments and Experimental Techniques, 2015, V. 58, Issue 4, pp. 510-514.

3. Tot'meninov E.M., Vykhodtsev P.V., Gunin A.V., Klimov A.I., Rostov V.V. Increase in the energy efficiency of a pulsedperiodic relativistic backward wave oscillator with a modulating resonant reflector // Technical Physics, 2014, V. 59, Issue 3, pp. 428-433.

4. Kitsanov S.A., Korovin S.D., Klimov A.I., Rostov V.V., Tot'meninov E.M. Mechanically tuned relativistic backward wave oscillator // Technical Physics Letters, 2004, V. 30, Issue 8, pp. 619-621.

5. Tarakanov V.P. Multipurpose electromagnetic code KARAT // in Mathematical Modeling: Problems and Results, 2003. Moscow, Nauka, Russia, pp. 456-476. 